\title{
Evaluating the Efficacy of Garlic and Cranberry Extracts against E. coli causing Urinary Tract Infections
}

\author{
Zahra Khosravi
}

\begin{abstract}
Background: Escherichia coli (E. coli) is the major cause of community-based Urinary tract infections (UTI). Products from nature have been used in traditional medicine practice although with limited scientific validity. In this study garlic and cranberry extracts was assessed for its efficacy to inhibit $E$. coli growth. Materials and Methods: Crude garlic and cranberry extracts were obtained and E. coli was isolated from an infected urine sample. Two-fold serial dilution was carried out on the $E$. coli and crude extract inoculations. Incubation and plating was carried out for $24 \mathrm{hr}$ at $30^{\circ} \mathrm{C}$. Colony count was done using a digital Quebec Colony Counter. Results: A general trend of reducing number of average CFU with increasing concentration of both extracts was observed. Garlic was observed to be more potent than the cranberry extract even at the lowest concentration. Conclusion: This study establishes the merit of using garlic and cranberry extract as a household remedy for UTI caused by E. coli.
\end{abstract}

Key words: Urinary tract infections, Garlic extract, Cranberry extract, E. coli, Antibiotic resistance, Prophylactic treatment.

\section{INTRODUCTION}

Uncomplicated urinary tract infections (UTI) are the most common reason for the prescription of antibiotics. Uropathogenic Escherichia coli (UPEC) is the agent in about $90 \%$ of community acquired infections. ${ }^{1}$ UPEC is a gram-negative bacterium and among its four known phylogenetic groups, the B2 and D group are associated with most UTI. ${ }^{2}$ All bacteria have pili on their surface for adhesion. However, most strains of Escherichia coli (E. coli) also exhibit fimbriae; shorter hair like structures that also aid in adhesion to surfaces because of the adhesin protein. ${ }^{3}$ They are essential for UPEC to bind to the epithelial surface of the cervix as well as other mucus membranes and are either sensitive or resistant to D-mannose, which causes them to agglutinate. Type 1 fimbriae are mannose sensitive, whilst Proteus like fimbriae (P. Fimbriae) are mannose resistant, causing recurrent infections. ${ }^{4}$ An infection is thus induced when lectins on the surfaces of the fimbriae attach to the complimentary carbohydrates on the mucus membrane ${ }^{5}$ which helps the bacteria to colonize the urinary tract.

Modern medicine owes its development to its often-forgotten roots in ancient herbal medicine practice. However, increasing antibiotic resistance is now a global threat according to WHO. ${ }^{6}$ Thus, it is appropriate to invest more time investigating herbal medicines that exhibit antibiotic properties. This study was designed to evaluate whether resources found in nature have the potential use in medicine. Two natural products i.e, Vaccinum macrocarpon

Cite this article : Khosravi Z. Evaluating the Efficacy of Garlic and Cranberry Extracts against $E$. coli causing Urinary Tract Infections. BEMS Reports. 2020;6(2):22-7.
(Cranberries) and Allium sativum (Garlic) with wellknown antibacterial properties were shortlisted for this study.

Cranberry is scientifically known as Vaccinum macrocarpon and extract of cranberries is reported to exhibit antibacterial properties. This is because they contain fructose, that blocks type 1 fimbriae, in addition to proanthocyanidins which are condensed tannins that competitively inhibit the adhesive properties of $P$. fimbriae by binding to its tips. ${ }^{7} \mathrm{~A}$ study by Hisano et al. ${ }^{8}$ Indicated that when treated with $50 \mu \mathrm{g} / \mathrm{mL}$ cranberry extract, most strains of E.coli showed $75 \%$ reduction in adhesion to epithelial cells. The proanthocyanidins also changes the conformation of $E$. coli surface molecules and reduces the expression of P-Fimbriae. Cranberry juice is also significant for its low $\mathrm{pH}$ of 2.5 , due to organic acids such as malic, quinic and shikmic acid which all create a hostile environment for the growth of gram-negative bacteria. ${ }^{9}$ Quinic acid can metabolize into hippuric acid, which has strong antibacterial properties. $^{10}$

Allium sativum or garlic contains allicin, a flavor compound with several pharmacological properties including antibacterial efficacy. ${ }^{11}$ Allicin inhibits many physiological processes in micro-organisms such as RNA synthesis and lipid biosynthesis. ${ }^{11}$ Allicin also inhibits the incorporation of acetate into fatty acid biosynthesis, as it inhibits acetyl-CoA synthase and this is significant because in bacterial systems, all enzymes, which produce acetyl-CoA

\section{0} access article distributed under the terms of the Creative Commons Attribution 4.0 International license. 
from acetate, are inhibited by allicin in a reversible manner through noncovalent binding. ${ }^{11}$ The antibacterial effect of garlic is also attributed to the inhibition of thiol containing enzymes in microorganisms. ${ }^{12}$

Based on the available literature, the research question to be evaluated was to what extent are various concentrations of Vaccinum macrocarpon (Cranberries) and Allium Sativum (Garlic) extract are effective in inhibiting the growth of $0.5 \mathrm{McF}$ arland standard E. coli suspensions when incubated for $24 \mathrm{hr}$ at $30^{\circ} \mathrm{C}$ and does it provide evidence for aiding in the treatment of UTI? Hence the aim of this study was to 1) To test whether pure extracts of $V$. macrocarpon and A. sativum decrease the colonies of E. coli and 2) To determine which of the two extracts are most effective after a 24-hr incubation period, by comparing its minimum inhibitory concentration (MIC).

\section{MATERIALS AND METHODS}

Preliminary experiment: Prior to determining the usage of garlic and cranberry extracts, a preliminary experiment for comparative evaluation of four natural product extracts was performed, to assess which was most effective at decreasing colonies (CFU) of E. coli after $24 \mathrm{hr}$ of incubation, when inoculated on agar plates. Pure extracts were vortexed with a solution of 0.5 McFarland standard E. coli suspended in nutritious ID broth and $0.01 \mathrm{ml}$ were inoculated right away on sheep blood and EMB (eosine methylene blue) agar. Dishes were incubated for $24 \mathrm{hr}$ at $30^{\circ} \mathrm{C}$.

Results after immediate inoculation:

$\begin{array}{ccc}\text { Extract/agent } & \text { Sheep blood agar } & \text { EMB agar } \\ \text { Garlic } & { }^{*} \text { TMTC } & \text { TMTC } \\ \text { Ginger } & \text { TMTC } & \text { TMTC } \\ \text { Cranberry } & \text { TMTC } & \text { TMTC } \\ \text { Manuka Honey } & \text { TMTC } & \text { TMTC } \\ \text { E.Coli suspension } & \text { TMTC } & \text { TMTC }\end{array}$

TMTC = Too many to count, an acceptable range is 30-300 colonies, due to statistical reasons (Escalano-Arranz et al. 2010).

${ }^{*}$ Colonies forming units (CFU) were to be counted with a digital colony counter however it was obvious to the naked eye that they surpassed that range.

For control, E. coli suspensions of 0.5 turbidity were inoculated as well so that comparisons could be made between the number of colonies. On questioning why this was, it was decided to leave $0.01 \mathrm{ml}$ solutions containing the pure extracts, $E$. coli and ID broth into the incubator for $24 \mathrm{hr}$ at $30^{\circ} \mathrm{C}$ and then inoculate onto agar dishes incubated for 24 $\mathrm{hr}$ at $30^{\circ} \mathrm{C}$ again $(48 \mathrm{hr}$ total). This allowed the E. coli and antibacterial reagents in extracts to have the proper environment and sufficient time to react together and thus display optimal results.

Results after incubation of solution:

The results indicate that garlic and cranberry extract were most effective. Hence these two extracts were shortlisted for further investigation. A two-fold serial dilution of these extract was used to determine which concentration is most effective at inhibiting bacterial growth.

$\begin{array}{ccc}\text { Extract/agent } & \text { Sheep blood agar } & \text { EMB agar } \\ \text { Garlic } & 0 & 0 \\ \text { Ginger } & \text { TMTC } & \text { TMTC } \\ \text { Cranberry } & 101 & 97 \\ \text { Manuka Honey } & \text { TMTC } & \text { TMTC } \\ \text { E.Coli suspension } & \text { TMTC } & \text { TMTC }\end{array}$

A notable qualitative observation made was that the gram-negative nature of $E$. coli was more pronounced on EMB agar and therefore colonies were more distinguishable, assisting the device in giving accurate results.

\section{Variables used}

Independent variable: Decreasing the concentration of pure extracts of garlic and cranberry from $50 \%$ to $25 \%$ to $12.5 \%$ to $6.25 \%$ and to $3.125 \%$ using a serial two-fold dilution.

Dependent variable: Number of colonies formed when $0.01 \mathrm{ml}$ of $E$. coli and varying concentrations of garlic and cranberry extracts are inoculated for $24 \mathrm{hr}$, applied on EMB agar plates and then incubated for $24 \mathrm{hr}$ at $30^{\circ} \mathrm{C}$, measured using a colony counter.

\section{Controlled variables (detailed in Table 1):}

- Same type of bacterium used - E. coli

- E. coli suspensions with nutrient broth adjusted to a $0.5 \mathrm{McF}$ arland turbidity standard previous to inoculation, using a turbidimeter

- Inoculations of extracts and E. coli will be incubated for $24 \mathrm{hr}$ before being spread on agar plates for counting of colonies.

- Stock solutions of extracts are all 'crude', with no disinfectants such as ethanol applied.

- The volume of inoculations spread on EMB agar plates - $0.01 \mathrm{ml}$

- Type of agar for streaking and plating (EMB) and broth solution used (ID broth)

- Conditions for incubation of agar plates with E. coli and extract solution $-24 \mathrm{hr}$ at $30^{\circ} \mathrm{C}$

\section{Microorganism}

Escherichia coli isolated from infected urine sample was used. The E. coli sample was sourced from the pathology laboratory, Iranian Hospital.

Chemical reagents and natural substances: BD Phoenix ID Broth (3 vials), Garlic (200.00 $\pm 0.01 \mathrm{~g})$, Cranberry $(200.00 \pm 0.01 \mathrm{~g})$ and EMB (eosin methylene blue) agar plates (50 numbers) were used in this study. Each 1 liter of BD Phoenix ID Broth (Funke et al. 2004) consisted of Potassium chloride $(7.5 \mathrm{~g})$, Calcium chloride $(0.5 \mathrm{~g})$, Tricine glycine $(0.895 \mathrm{~g})$, Polysorbate $80(0.025 \%)$ and distilled water (qs). Besides these, sterile $100 \mathrm{ml}$ glass vials with caps, Sterile $10 \mu \mathrm{L}$ disposable loops, Micropipette, Biosafety cabinet, Digital incubator, Densitometer, Digital colony counter, Blender, Knife, Centrifuge machine and Vortexer was used in this study.

Sterilization: All none sterilized equipment was sterilized with rubbing alcohol to decrease contamination previous to usage.

Isolation of $\boldsymbol{E}$. coli and culturing: $E$. coli was isolated from a sample of infected urine by a qualified and professional laboratory technician and streaked onto EMB agar plates, using a sterile swab. The sample was incubated for $24 \mathrm{hr}$ at $30^{\circ} \mathrm{C}$ before being used for experimentation.

Preparation of extracts: Garlic and cranberry were obtained from a local supermarket. They were both rinsed with water to remove any dirt and allowed to air dry at room temperature $\left(25^{\circ} \mathrm{C}\right)$ for three hr. Garlic bulbs were peeled and cranberries destemmed and $500 \mathrm{~g}$ were weighed using digital weighing scales for high precision.

Weighed garlic bulbs were placed into a sterilized blender and blended to a puree consistency. Liquid juice from the puree was squeezed out using a mesh cloth, into a sterilized glass container. Gloves were worn while doing this. Pure cranberry juice was prepared using this method as well. $20 \mathrm{ml}$ of each was then transferred into individual sterilized containers using droppers. Containers were refrigerated at $18^{\circ} \mathrm{C}$ overnight, as 'humidity and increase in temperature cause degradation in the antibiotic properties of natural extracts' due to denaturation. ${ }^{13}$ No solvents (such as alcohols) were used for the preparation of the extract as this investigation 
aimed to report results about whether these plant materials can be used at home readily for the treatment of UTI, for translation of the study results to real life application.

Preparation of inoculums: Densitometer was first plugged in and allowed to calibrate. All inoculums were prepared under the space of a biosafety cabinet while wearing gloves. A sterile cotton swab was used to collect bacterial colonies from the initial culture plate of $E$. coli and suspended into a vial of ID broth by rapid shaking of the swab. Once the material was off the swab, the vial was closed and vortexed for $7 \mathrm{sec}$, so E. coli sample was fully integrated into broth solution. The turbidity was measured by a densitometer to a $0.5 \mathrm{McF}$ arland standard, equivalent to approximately $1.5 \times 10^{8} \mathrm{CFU} / \mathrm{ml}^{14}$ Adjustments were made if reading was off by creating a new sample entirely (when reading was higher) and adding more $E$. coli with a sterile swab (when reading was lower). ${ }^{15,16}$ Three separate inoculums of 0.5 standard pure E. coli were prepared the same way for control (no extract), garlic extract and cranberry extract.

Serial dilution: Separate glass vials were labeled and 0.002, 0.004,0.008, 0.016 and 0.032 and $0.100 \mathrm{ml}$ of the 0.5 standard $E$. coli inoculum was added in each using a micropipette with a new tip each time to decrease contamination. $100 \mathrm{~mL}$ of cranberry extract was centrifuged at $320 \mathrm{rpm}$ for a few seconds so that denser particles would sink, allowing extract to be measured out more accurately. $0.1 \mathrm{~mL}$ of stock cranberry extract was added to vial labeled ' 2 ' and a serial two-fold dilution was carried out, going from 1:2, 1:4, 1:8, 1:16 and 1:32, ratio of standard E. coli culture:natural extract concentration. Each vial was closed and vortexed for $7 \mathrm{sec}$ to ensure both solutions are mixed. The same method was carried out for the serial dilution of garlic extract, except dilutions of 1:64 and 1:128 were included as well based on the preliminary experiment which showed garlic had a much stronger tendency to inhibit the growth of $E$. coli. Serial dilution was done under the sterile conditions of the biosafety cabinet, while wearing latex gloves. All samples were then incubated for $24 \mathrm{hr}$ at $30^{\circ} \mathrm{C}$, for the antimicrobial agents to affect the bacterial growth in-vitro.

Plating: Plating was done on EMB agar plates under the sterile environment of the biosafety cabinet while wearing gloves. $0.01 \mathrm{ml}$ of each inoculum was spread on each separately labeled agar plates with their respective concentrations, using a sterile $0.01 \mathrm{ml}$ loop. If the sample

Table 1: Controlled variables used.

Controlled (for all trials and concentrations of extracts)

Type of bacterium used - E. coli

Type of agar used for streaking and plating - EMB agar and type of nutrition broth - ID broth

Turbidity of E. coli and nutrient ID broth previous to inoculation - 0.5 McFarland turbidity standard.

Volume of $E$. coli and ID broth suspension in each vial before dilution $-0.1 \mathrm{~mL}$

Amount of time and temperature that the extracts of different concentrations mixed with the E. coli suspensions are inoculated and incubated for before plating - $24 \mathrm{hr}$ at $30^{\circ} \mathrm{C}$

Amount and conditions for incubation for plating of each inoculation $-0.01 \mathrm{~mL}$ for 24 $\mathrm{hr}$ at $30^{\circ} \mathrm{C}$

Preparation of pure (crude) extracts of garlic and cranberry should not be done with any extra additives such as ethanol.

Method of control

Possible effect on results

E. coli will be isolated from urine sample of patient with urinary tract infection using gram staining ( $E$. coli is gram negative, so it will not retain the violet dye) and morphological analysis.

The E. coli will then be cultured again on a separate EMB plate and will be used for inoculations.

EMB agar and ID broth are readily available for purchase and will be used.

Sample of $E$. coli will be swabbed from the culture and vortexed with a solution of ID broth and then placed into a turbidimeter. If it is less than 0.5 , it will be effectively adjusted by the addition of more $E$. coli with a swab and if it is more, a new sample will be made with a new vial of ID broth.

$100 \mu \mathrm{L}$ will be added using a micropipette with a new tip each time to decrease the amount of contamination.

Once the serial two fold dilution is performed for each of the pure extracts and added to respective vials each containing $100 \mu \mathrm{L}$ of the E. coli suspension and vortex, they will be incubated for $24 \mathrm{hr}$ at $30^{\circ} \mathrm{C}$, to allow the bacteria to react and grow in the presence of the antibacterial agents sufficiently.

A sterile disposable $10 \mu \mathrm{L}$ loop will be used to obtain samples from each dilution and then streaked onto EMB agar.

Pure extracts will be prepared by blending fresh cranberry and garlic and then straining through mesh cloth to collect the liquid juice.
Different types of bacteria respond differently to antibiotic agents, therefore to ensure the accuracy of the results, only one type of bacterium must be used to test the antibiotic effects of garlic and cranberry extracts. E. coli specifically have $P$. fibriae which are targeted by antibiotic agents in cranberries.

Different types of agar and broths have different conditions such as $\mathrm{pH}$ and type of nutrients and so this needs to be controlled in order to keep other factors that affect the growth of $E$. coli constant and improve accuracy of the results.

As turbidity is an estimate for the amount of bacterium in a sample ( 0.5 is equivalent to approximately $1.5 \times 10^{8}$ $\mathrm{CFU} / \mathrm{ml}$ ), it needs to be kept constant before inoculation with samples of garlic and cranberry extracts in order to compare between the two in terms of their effectiveness. A higher amount of bacteria would require a higher concentration of antibiotic agents.

Since it is a serial dilution of the extracts, a set amount of diluent (E. coli suspension) must be used, in order for accurate concentrations of extracts to be made for experimentation.

This needs to be controlled so that results are reliable for comparison and that the colonies of bacteria formed after plating are accurate for counting. If the time and temperature for incubation are not controlled, the growth rate of bacteria will be affected as they have a set of optimum conditions.

This controls the amount of bacteria set on each plate and thus makes results reliable for comparison, because a higher volume would contain more bacteria which would then affect the accuracy of the results. $24 \mathrm{hr}$ and $30^{\circ} \mathrm{C}$ are optimum growth conditions for $E$. coli.

The reason this is being done is because the extracts have to be as natural as possible, in order to be able to evaluate how effective they are at inhibiting the growth of bacteria in real life situations such as a UTI. 
failed to reside on the plate, a new loop was used to take the sample and spread on a new agar plate. The original inoculums were plated as well, to provide as a control measure for comparison. All plates were incubated for $24 \mathrm{hr}$ at $30^{\circ} \mathrm{C}$.

Colony count: After incubation, colony count was done for each plate using a digital Quebec Colony Counter. Although increased accuracy would be obtained if a spectrophotometer, especially considering the small changes that may occur in the growth of $E$. coli with different concentrations and precision would also be improved. However it was not available. Hence CFU (colony forming units) were counted twice for each plate, for accuracy to be increased. Above method was repeated for 20 trials, with controlled variables and safety measures fully acknowledged. All procedures took place in a sterile clinical laboratory under the supervision of a professional lab technician, in a biosafety cabinet. All materials disposed of safely in the appropriate biosafety bin.

\section{Data processing and analysis}

A more appropriate way to report results is calculating $\mathrm{CFU} / \mathrm{ml}$ for each $\%$ concentration, using the equation:

$\mathrm{CFU} / \mathrm{ml}=\frac{\mathrm{CFU}(\text { colonies counted })}{\text { Volume plated } \mathrm{x} \text { dilution factor }}$

Where volume plated $=0.01 \mathrm{ml}$

Where dilution factor $=\frac{\text { Final volume }}{\text { Sample volume }+ \text { Diluent volume }}$

Thus, dilution factor $=0.1000 .100+0.100=0.500$ and this is held constant for each dilution. Calculations was performed using Microsoft Excel

The data was analyzed using repeated measures ANOVA for cranberry as there is more than two concentrations being compared and paired $\mathrm{t}$-test for garlic since two concentrations are being compared, using SPSS software for determination of whether differences in the means was significant.

\section{RESULTS}

Mauchly's Test of Sphericity was used to assess the condition of equality between variances of all cranberry concentrations. The assumption of sphericity has been met since $p>0.05$ (0.470); variances of differences between all concentrations within cranberry extracts are not equal, hence assumption has not been violated and was further assessed by repeated measures ANOVA.

The average number of CFU observed in the each of the extract treated group is summarized in Table 2. A general trend of reducing number of average $\mathrm{CFU}$ with increasing concentration of both extracts was

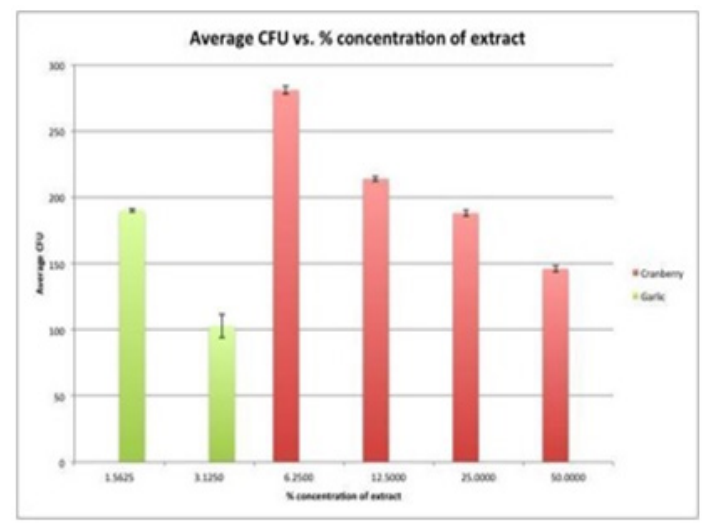

Figure 1: Concentration dependent effect of the extract on average number of CFU. Data is presented as Mean \pm SD. observed. Garlic is observed to be more potent than the cranberry extract even at the lowest concentration (Figure 1). Nevertheless both the extracts tested were observed to effectively reduce the number of $E$. coli colonies (Figure 1). The CFU/ml of each extract was also observed to be superior with garlic extract (Table 3). A concentration dependent effect of cranberry extract was observed on the $E$ coli $\log \mathrm{CFU} / \mathrm{ml}$ levels (Figure 2). Garlic extract had higher potency than the cranberry extract, as no colonies were observed in the garlic extract above $3.1250 \%$ concentration.

The difference in the means of CFU for cranberry extracts was statistically significant $(\mathrm{F}(3,54)=18524.143, p=0.000)$. The post-hoc analysis revealed that the results for all cranberry concentrations (that resulted in a decrease of CFU) was statistically significant $(p<0.05)$. The difference in the means of CFU for garlic extracts was statistically significant $(p<0.05)$ as well.

\section{DISCUSSION}

The garlic and cranberry extract showed significant antimicrobial efficacy against clinical isolates of $E$. coli causing urinary tract infection. Thus this study establishes the merit of using garlic and cranberry extract as a household remedy for UTI caused by E. coli. The antimicrobial efficacy against clinical isolates of $E$. coli of garlic extract was observed to be superior to the cranberry extract. Garlic was more potent, as no colonies were formed with concentrations up until $3.1250 \%$. Both cranberry and

Table 2: Average number of CFU vs concentration of extracts.

\begin{tabular}{ccc} 
& \multicolumn{2}{c}{ Average number of CFU } \\
\hline \% Concentration of extracts & Cranberry & Garlic \\
50.0000 & $146 \pm 2$ & $* * 0$ \\
25.0000 & $188 \pm 2$ & $* * 0$ \\
12.5000 & $214 \pm 2$ & $* * 0$ \\
6.2500 & $281 \pm 3$ & $* * 0$ \\
3.1250 & $*_{-}$ & $103 \pm 9$ \\
1.5625 & $*_{-}$ & $190 \pm 1$
\end{tabular}

Data is represented as Mean \pm SD. ${ }^{*}$ Number of colonies $>300$, therefore too inaccurate to be counted. ${ }^{* *}$ No colonies present for counting. Average calculated by where $n=20$ (total number of trials). $0.0078125 \%$ ( $1: 128$ ) concentration omitted because results are not in 30-300 colony range for each extract.

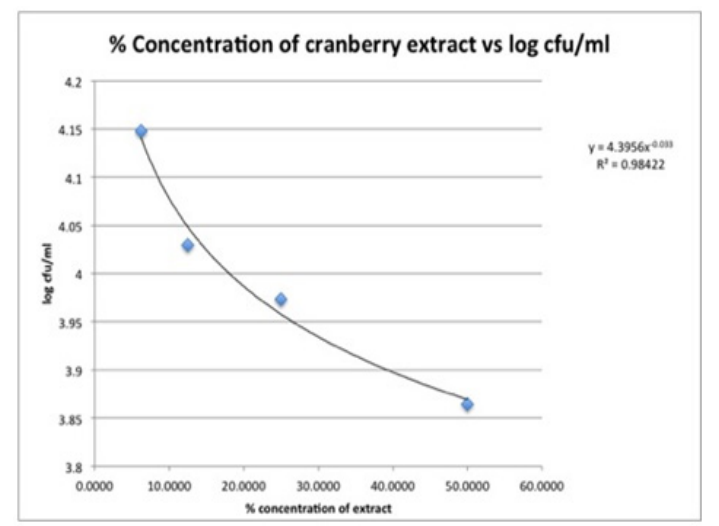

Figure 2: Concentration dependent effect of the cranberry extract on E. coli $\log \mathrm{CFU} / \mathrm{ml}$. 
Table 3: CFU/ml of each extract vs $\%$ concentration.

\begin{tabular}{cccccrr}
\multicolumn{7}{c}{ CFU/ml } \\
& \multicolumn{7}{c}{ \% Concentration of extract } \\
& 1.5625 & 3.1250 & 6.2500 & 12.5000 & 25.000 & 50.0000 \\
Cranberry & - & - & 14050.00 & 10710.53 & 9410.53 & 7321.05 \\
Garlic & 9513.16 & 5150.00 & - & - & - & -
\end{tabular}

garlic extracts reduced the CFU of E. coli after a 24-hr incubation period, however, this was only effective for cranberry up to a concentration of $6.125 \%$, as for following lower concentrations, the number of colonies formed were too high to count accurately. Although this study did not evaluate the combined effects of cranberry and garlic extracts, this may be evaluated in future studies to assess any potential pharmacological synergism. The reason for garlic being more effective is probably because of its organo sulfur compounds which directly target processes that are required for the growth of E. coli. Garlic can have better efficacy by effectively penetrating phospholipid bilayers directly and binding to enzymes such as acetyl-CoA and inhibiting them which in turn causes immense changes to cell viability, ${ }^{11}$ in contrast proanthocyanidins in cranberries create a hostile environment and decrease the expression of fibriae on E. coli, preventing them from binding to other membranes. ${ }^{7}$

In this study the CFU were counted manually, which is a less accurate technique than automated cell counters. This may perhaps be the reason for observing a large deviation in the results of CFU numbers. Use of automated cell counters may improve the statistical precision of this study, which should be considered by any group interested in independently replicating this study findings. Although the concentration of garlic extract required to reduce the E. coli CFU was lower, a precise MIC cannot be established as the range of dilutions is neither large nor precise enough for an accurate conclusion to be made. Nevertheless the concentration dependent effects of both extract on effectively reducing the $\mathrm{CFU} / \mathrm{ml}$ of clinical isolate of $E$. coli, merits their use as a household remedy for UTI. At high concentrations (25\% and 50\%) pure natural extracts of cranberry are effective at reducing the number of $E$. coli colonies but, garlic has higher potency (with colonies that appeared at $1.5635 \%$ and $3.1250 \%$ concentrations only). Cranberries contain proanthocyanidins ${ }^{7}$ which are of low $\mathrm{pH}$ and exhibit anti-adhesive properties and garlic contains allicin, inhibiting physiological processes such as RNA synthesis in microorganisms. However, in accordance with the data collected, effectiveness decreases with decreasing concentration of pure extracts and similar results were reported in previous studies done by Lu et al. and Nicolosi et al.

The two-fold dilution used in this study limits its use in the estimation of MIC for each extract. Nevertheless as more concentrations were tested, a line of best fit could have defined an asymptote, representing the maximum number of $\mathrm{CFU}$ reached with each extract. This is especially remarkable because of the significant differences in the efficacy of cranberry and garlic extracts. Future studies should look at assessing a tenfold dilution of the extracts. A ten-fold dilution would allow for a higher range of concentrations to be tested and for results regarding the efficacy of each extract to be more precise. The purity of the extracts used in this study also has its limitations. Even though they were prepared with sterile equipment and washed with distilled water, there might have been some contamination due to the extracts not being purified by methods such as high-performance liquid chromatography. ${ }^{18}$ This may have contributed to larger variances in the study results. Another source of variance in the study results is from the byproducts produced by the bacteria's in the culture which can potentially influence the pharmacological efficacy of the extracts. This can happen when the extracts act as a source of nutrition for the bacteria. Hence using the specific purified antibiotic agents in each extract rather than the whole extract itself, may be warranted in future studies. A further limitation of the methodology was not using a positive standard in comparing the relative efficacy of the extracts used. Hence future studies in addition to including a positive standard should also aim to assess potential synergistic effects of these extracts.

Although results of this investigation have answered the research question proposed, it is important to consider how applicable they are in real-life. Firstly, if considering pure extracts will be consumed, chances of garlic being consumed raw rather than being added into cooking is low. But, organosulfur compounds become denatured when it is cooked and so, antibiotic activity is reduced, decreasing its efficacy. Secondly, it cannot be concluded that the two extracts can be used to treat UTIs as they are still much less effective than clinical antibiotics for short term treatments. ${ }^{8}$ Although, they could potentially be used as prophylaxis in the long-term to be deemed effective for recurrent UTIs ${ }^{8}$ and do not pose the risk of antibiotic resistance. A study by Behzadi et al. ${ }^{19}$ indicated that there may be many other bacteria present in a UTI such as K. pneumoniae which means that the results of this study should be further supported by additional research before its wider application in real life. A considerable extension would be to test the antibiotic effect of cranberry and garlic on various other strains of bacteria, to optimally assess their efficacy.

\section{CONCLUSION}

Overall, this study has resulted in highlighting the antimicrobial efficacy of cranberry and garlic extracts at different concentrations on clinical isolate of $E$. coli associated with UTI. The positive findings from this study merits further studies to address the study limitations described above.

\section{ACKNOWLEDGEMENT}

The author would like to express her gratitude to Mrs. Raja Kassem of Emirates International School Jumeirah, and Dr. Arun Kumar of Univesity College Dublin for their guidance in making this publishing possible.

\section{CONFLICT OF INTEREST}

The author declares none.

\section{ABBREVIATIONS}

TMTC: Too many to count; CFU: Colony forming unit; EMB: Eosin methylene blue; MIC: Minimum inhibitory concentration.

\section{REFERENCES}

\footnotetext{
1. Hidalgo G, Chan M, Tufenkji N. Inhibition of Escherichia coli CFT073 fliC expression and motility by cranberry materials. Appl Environ Microbiol. $2011 ; 77(19): 6852-7$.
} 
2. Hagberg L, Jodal U, Korhonen TK, Lidin-Janson G, Lindberg U, Svanborg EC. Adhesion, hemagglutination and virulence of Escherichia coli causing urinary tract infections. Infect Immun. 1981;31(2):564-70.

3. Wagenlehner FM, Hoyme U, Kaase M, Funfstuck R, Naber KG, Schmiemann G. Uncomplicated urinary tract infections. Dtsch Arztebl Int. 2011;108(24):415-23.

4. Calbo E, Romani V, Xercavins M, Gomez L, Vidal CG, Quintana S, et al. Risk factors for community-onset urinary tract infections due to Escherichia coli harbouring extended-spectrum beta-lactamases. J Antimicrob Chemother. 2006;57(4):780-3

5. Svanborg-Eden C, Jodal U. Attachment of Escherichia coli to urinary sediment epithelial cells from urinary tract infection-prone and healthy children. Infect Immun. 1979;26(3):837-40.

6. World Health Organization. Antiobiotic resistance. 2018.

7. Nicolosi D, Tempera G, Genovese C, Furneri PM. Anti-Adhesion Activity of A2type Proanthocyanidins (a Cranberry Major Component) on Uropathogenic $E$. coli and P. mirabilis Strains. Antibiotics. 2014;3(2):143-54.

8. Hisano M, Bruschini H, Nicodemo AC, Srougi M. Cranberries and lower urinary tract infection prevention. Clinics. 2012;67(6):661-8.

9. Ghosh S, Pawar H, Pai O, Banerjee UC. Microbial transformation of quinic acid to shikimic acid by Bacillus megaterium. Bioresources and Bioprocessing. 2014;1(1):7.

10. Jensen HD, Struve C, Christensen SB, Krogfelt KA. Cranberry Juice and Combinations of its Organic Acids Are Effective against Experimental Urinary Tract Infection. Front Microbiol. 2017;8:542.
11. Focke M, Feld A, Lichtenthaler K. Allicin, a naturally occurring antibiotic from garlic, specifically inhibits acetyl-CoA synthetase. FEBS Lett. 1990;261(1):106 8.

12. Lu X, Rasco BA, Jabal JM, Aston DE, Lin M, Konkel ME. Investigating antibacterial effects of garlic (Allium sativum) concentrate and garlic-derived organosulfur compounds on Campylobacter jejuni by using Fourier transform infrared spectroscopy, Raman spectroscopy and electron microscopy. Appl Environ Microbiol. 2011;77(15):5257-69.

13. Yano Y, Satomi M, Oikawa H. Antimicrobial effect of spices and herbs on Vibrio parahaemolyticus. Int J Food Microbiol. 2006;111(1):6-11.

14. JA W. McFarland Standard For in vitro use only. 2014.

15. Funke G, Funke-Kissling P. Use of the BD PHOENIX Automated Microbiology System for direct identification and susceptibility testing of gram-negative rods from positive blood cultures in a three-phase trial. J Clin Microbiol. 2004;42(4):1466-70.

16. AT. Preparation of McFarland Turbidity Standards. 2016.

17. JP. Antimicrobial Effect of Cranberry Powder on E. coli ATCC 25922 in Apple Cider and Ground Beef. The Graduate School: University of Wisconsin-Stout. 2010.

18. Libre T. High Performance Liquid Chromatography. 2019.

19. Behzadi P, Behzadi E, Yazdanbod H, Aghapour R, Akbari CM, Salehian OD. A survey on urinary tract infections associated with the three most common uropathogenic bacteria. Maedica. 2010;5(2):111-5.

Cite this article : Khosravi Z. Evaluating the Efficacy of Garlic and Cranberry Extracts against E. coli Causing Urinary Tract Infections. BEMS Reports. 2020;6(2):22-7. 